\title{
Comparative Analysis of Inhomogeneity Degree of Magnetic Field of Polygradient Magnetic Separators for Purification of Bulk Materials
}

\author{
Iryna Shvedchykova, Julia Romanchenko, Inna Nikitchenko \\ Volodymyr Dahl East Ukrainian National University \\ Severodonetsk, Ukraine \\ ishved@i.ua, romanchenkojulia@i.ua, inna.mia.lg@gmail.com
}

\begin{abstract}
The paper deals with the main approaches for estimation of homogeneity degree of magnetic field in polygradient medium of magnetic separator. As an indicator of the inhomogeneity of field was accepted inhomogeneity coefficient. It shows how many times the strength of field at research point differs from the strength of homogeneous field. Numerical simulation of distribution of the magnetic field in interpolar working zones in a two-dimensional formulation for six synthesized structures. The plate in form of rectangular triangle was adopted as an elementary monostructure was carried out. Comparative estimation of inhomogeneity degree of magnetic field along characteristic lines of working zones of synthesized structures of magnetic separators polygradient medium has been made. For field inhomogeneity research as characteristic lines were accepted lines that connect the vertices of the opposing triangular plates of the matrix. The results of research can be used to choose the rational structure of polygradient medium and geometric dimensions of its plate elements.
\end{abstract}

Keywords-magnetic separator; magnetic field; structural synthesis; inhomogeneity coefficient; polygradient medium.

\section{INTRODUCTION}

In agriculture and construction polygradient magnetic separators are used to improve cleaning efficiency of bulk materials (for example, flour, gypsum, cereals) from ferromagnetic particles of $0,05 \mathrm{~mm}$ or less that formed as a result of wear of friction parts of processing equipment. Polygradient magnetic separators are also widely used as ecological equipment for purification of solid waste, gas emissions and wastewater [1, 2]. In such separators, raw material passes through a polygradient medium consisting of ferromagnetic objects (plates, spheres, cylinders, rods) located in matrix (cassette) situated in magnetic field of separator. In zone of arrangement of ferromagnetic bodies, spatially inhomogeneous magnetic fields with a high gradient of field strength are created, what makes possible to develop bigger magnetic forces necessary for extraction of finely dispersed metallic inclusions.

Structure of polygradient medium (shape, mutual arrangement of ferromagnetic elements) and its geometric dimensions cause significant influence on strength distribution of magnetic field [3-5]. Therefore, choice of such designs of polygradient separator matrices that provide the highest magnetic-field inhomogeneity in interpolar working gaps and, consequently, high force characteristics of devices is actual and important task.

\section{PROBlEM STATEMENT AND RESEARCH METHODOLOGY SUBSTANTIATION}

Until recently, the choice of structure of separators polygradient matrices was carried out mainly empirically. With the development of methodological instruments of structural-system approach became possible a directed search and synthesis of new variants of polygradient medium structures [6]. Thus, using genetic modeling and consistent application of mirror, portable, central and sliding symmetry operations six structural variants (compositions) $\left(S_{1}, S_{2}, \ldots, S_{6}\right)$ of plate-shaped polygradient medium were received.

Plate medium (sets of plates) of matrices of polygradient separators refer to contactless type of polygradient medium with high magnetic resistance of interpolar working volumes, which requires a significant increase of the magnetic field strength to save the extracting force. At the same time, advantages of plate medium include easy cleaning from the extracted inclusions due to sufficiently wide gaps between the elements, which causes their wide application for purification of finely divided bulk materials from ferromagnetic impurities.

The choice from set $\left(S_{1}, S_{2}, \ldots, S_{6}\right)$ of synthesized structures the rational structures of the polygradient medium and geometric dimensions of its plate elements at which maximum heterogeneity of magnetic field in the working zone is achieved is the main issue of further research. This problem can be solved by method of variant calculations with a variation of matrix structure and its geometric parameters, i.e. by carrying out a computational experiment.

By computational experiment, we mean any use of mathematical model of technical device, which allows to find (as in a usual experiment) a certain interconnection of parameters of device or processes accompanying its operation. At the same time confirmation of results of the computational experiment by results of experiments on full-scale samples or physical models is redundant, since the computational experiment is based on a complete mathematical model of physical processes in the object. It should be noted that 
multivariate calculations are a difficult process, as for each of the options new models of magnetic systems must be built.

When carrying out a computational experiment the estimation of homogeneity degree of magnetic field in polygradient medium of synthesized structures can be performed using three main approaches:

1. By comparing the indicators of field inhomogeneity in similar points under condition of geometric similarity of working zones.

2. By comparing the indicators of field inhomogeneity along characteristic lines. Such approach as a whole is invariant to similarity transformations however the choice of characteristic lines depends on the location of zones of field inhomogeneity in working gaps. It is expediently to place similar points along characteristic lines for geometrically similar working zones.

3. By estimating of spectrum of field inhomogeneity in plane of working zone, for example, in accordance with the algorithm proposed in [7]. A feature of this approach is invariance to similarity transformations and location of zones of field inhomogeneity.

The purpose of this paper is to choose rational configuration of plate-shaped polygradient medium as well as its main active dimensions by comparative estimation of magnetic field inhomogeneity degree along the characteristic lines of working zones of synthesized structures.

\section{DESCRIPTION OF COMPUTATIONAL EXPERIMENT}

\section{A. Development of computer models of working zones of synthesized structures}

For computational experiment the licensed program package ELCUT 5.6 was used. ELCUT objects provide creation of new problem, description of its geometric model, construction of a finite element grid, solution of the problem and analysis of the results of solution in interactive view by graphical interface.

In order to determine the optimal configuration of polygradient medium and its active dimensions a numerical simulation of distribution of the magnetic field in interpolar working zones in a two-dimensional formulation was carried out. Simulation was carried out for six synthesized structures $\left(S_{1}, S_{2}, \ldots, S_{6}\right)$ in which plate in form of rectangular triangle was adopted as an elementary monostructure.

Fig. 1 shows the configurations of research working zones of magnetic systems and distribution of magnetic field in them. The synthesized structures $\left(S_{1}, S_{2}, \ldots, S_{6}\right)$ were obtained using the following symmetry operations: $S_{1}-$ mirror symmetry about the $O Y$ axis; $S_{2}$ - mirror symmetry about the $O Y$ axis with slip; $S_{3}-$ mirror symmetry about the axes $O X$ and $O Y ; S_{4}$ - mirror symmetry about the axes $O X$ and $O Y$ with slip; $S_{5}$-central symmetry; $S_{6}-$ central symmetry with slip. Fig. 2 shows the main geometric dimensions of working zone, which were varied during research, on the example of the $S_{3}$ structure. Accepted designations: $\delta-$ an interpolar working gap corresponding to minimum distance between the plates; $\alpha$ - angle at the top of the pole tooth; $b$-base of the pole tooth; $a$-working width of the matrix.

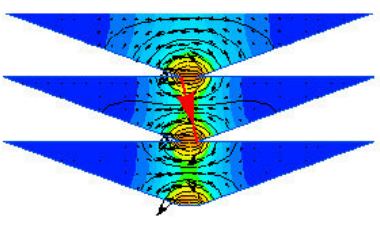

$S_{1}$

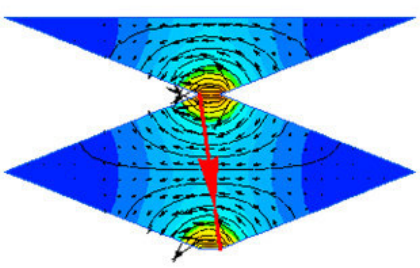

$S_{3}$

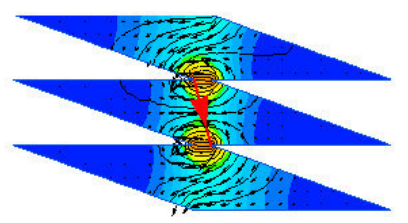

$S_{5}$

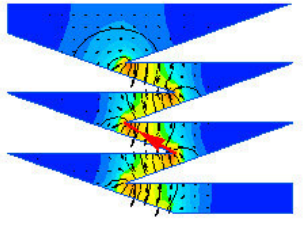

$S_{2}$

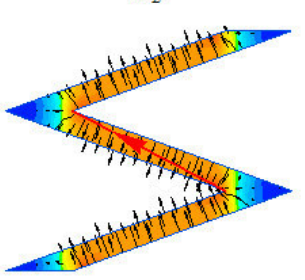

$S_{4}$

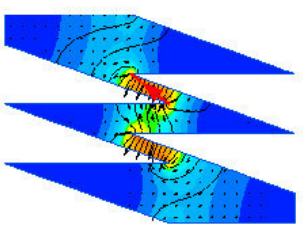

$S_{6}$
Fig. 1. Distribtion of magnetic field in the working air gap of the structures.

The following geometric criteria were established for the investigated regions: $X_{l}=b / a$ и $X_{2}=\alpha$. The ranges of variation of geometric criteria $X_{1}$ and $X_{2}$, that present practical interest, were: $X_{I}=b / a=0,2 \ldots 0,4 ; X_{2}=\alpha=0,22 \pi \ldots 0,44 \pi$. For description of physical properties of the problem limit conditions were set taking into account the relative magnetic permeability of air $\mu_{\mathrm{r}}=1$. Fig. 3 shows the geometric model of the working zone, on example of the $S_{4}$ structure.
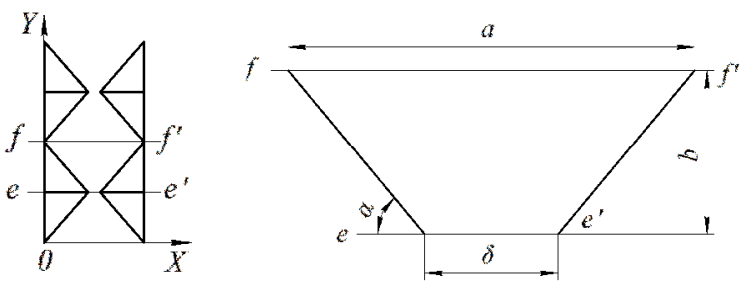

Fig. 2. Main geometric dimensions of working zone, on example of the $S_{3}$ structure.

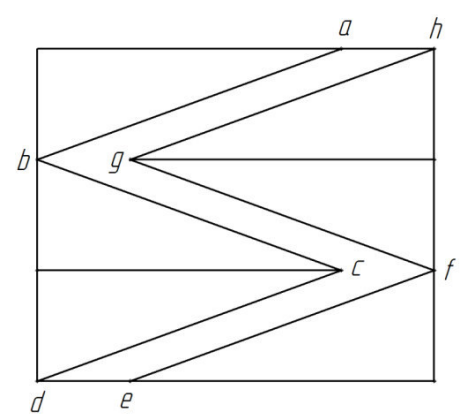

Fig. 3. The geometric model of the working zone, on example of the $S_{4}$ structure.

For the calculation zone limited by the contour abcdefgh, the following limit conditions were set. 
$a h$ - line of force with vector magnetic potential $\boldsymbol{A}=0$ (the Dirichlet condition). Such limit condition is used to indicate the complete extinction of the field at a remote border from source.

de - line of force for which the Dirichlet condition is also established. This condition determines behavior of normal component of induction on border. On segment $d e$ the value of vector magnetic potential $\boldsymbol{A}$ is given arbitrarily.

$a b, b c, c d, e f, f g, g h$ - borders (limits) corresponding to steel surfaces for which $H_{t}=0$ (homogeneous Neumann condition). Such limit condition is natural, since it is set by default on all those sides that form the outer border, where another limit condition is not obviously indicated.

For research of field inhomogeneity characteristic lines (highlighted in red in Fig. 1) that connects the vertices of the opposing triangular plates of the matrix were accepted. Characteristic lines pass through zones near the teeth with the highest heterogeneity of magnetic field.

\section{B. Results of computational experiment}

Along the characteristic lines in research working zones of the synthesized structures local values of the magnetic field strength $H$ were measured. As an indicator of the inhomogeneity of field accepted coefficient $k=H / H_{0}$, equals to the ratio of intensity $H$ in research point, that lying on the characteristic line, to the strength $H_{0}$ of the homogeneous field at the same value of the air gap $\delta$. Thus, the inhomogeneity coefficient $k$ shows how many times the strength of field at research point differs from strength of homogeneous field with unchanged dimension of air gap $\delta$. Table 1 shows the calculation results of inhomogeneity coefficient of field $k$ depending on geometric dimensions of the plates and for various configurations of polygradient medium. Measurements were carried out at geometric similarity criteria equals to $X_{1}=b / a=0,2 ; 0,3 ; 0,4 ; X_{2}=\alpha=0,22 \pi ; 0,33 \pi ; 0,44 \pi$.

Comparative analysis of calculated values of inhomogeneity coefficient of field $k$ showed that at $X_{2}=\alpha=0,44 \pi$ and any value of $X_{1}=b / a$ from range $X_{I}=b / a=0,2 \ldots 0,4$, magnetic field in working gaps has relatively low inhomogeneity degree. At $X_{2}=\alpha=0,44 \pi$, maximal value of inhomogeneity coefficient of field $k$ in zone that close to teeth was 1,75 (for the $S_{l}$ structure at $X_{I}=b / a=0,3$ ), and minimum value was 1,13 (for the $S_{2}$ structure at $\left.X_{l}=b / a=0,2\right)$. More interesting, from practical point of view, was analysis of inhomogeneity of field in synthesized structures $\left(S_{1}, S_{2}, \ldots, S_{6}\right)$ at $X_{1}=b / a=0,2 ; 0,3$ and $X_{2}=\alpha=0,22 \pi ; 0,33 \pi$.

At $X_{1}=b / a=0,2$, the highest inhomogeneity degree of field, and, consequently, the force characteristics of device provides the $S_{4}$ structure. Maximum value of coefficient $k$ in close-teeth zone for the $S_{4}$ structure was 2,57...2,7 (at $X_{2}=\alpha=0,22 \pi$ ) and $2,03 \ldots 2,05$ (at $X_{2}=\alpha=0,33 \pi$ ). Also in zone between teeth of the $S_{4}$ structure magnetic field approaches to homogeneous $(k \sim 1)$.
TABLE I. THE RESULTS OF INHOMOGENETEITY COEFFICIENT OF FIELD CALCULATION

\begin{tabular}{|c|c|c|c|c|c|c|}
\hline \multirow{2}{*}{$\begin{array}{l}\text { Code of } \\
\text { structure }\end{array}$} & \multicolumn{6}{|c|}{ Coordinates of measurement points in relative units } \\
\hline & 0 & 0,2 & 0,4 & 0,6 & 0,8 & 1,0 \\
\hline \multicolumn{7}{|c|}{$X_{I}=b / a=0,2, X_{2}=\alpha=0,22 \pi$} \\
\hline$S_{I}$ & 2,15 & 1,02 & 1,01 & 1,02 & 1,03 & 2,33 \\
\hline$S_{2}$ & 1,98 & 0,92 & 0,88 & 0,88 & 0,9 & 2,04 \\
\hline$S_{3}$ & 2,71 & 1,02 & 0,98 & 0,98 & 1,02 & 1,56 \\
\hline$S_{4}$ & 2,57 & 1,07 & 1,0 & 1,0 & 1,07 & 2,7 \\
\hline$S_{5}$ & 2,18 & 1,04 & 1,01 & 1,01 & 1,01 & 2,09 \\
\hline$S_{6}$ & 2,28 & 0,91 & 0,91 & 0,88 & 0,91 & 1,89 \\
\hline \multicolumn{7}{|c|}{$X_{I}=b / a=0,3, X_{2}=\alpha=0,22 \pi$} \\
\hline$S_{I}$ & 2,62 & 1,04 & 1,0 & 1,02 & 1,11 & 2,69 \\
\hline$S_{2}$ & 2,25 & 1,05 & 0,95 & 0,95 & 1,05 & 2,31 \\
\hline$S_{3}$ & 3,09 & 1,01 & 0,85 & 0,85 & 1,02 & 1,81 \\
\hline$S_{4}$ & 2,23 & 1,13 & 0,98 & 0,98 & 1,16 & 2,71 \\
\hline$S_{5}$ & 2,42 & 1,06 & 1,01 & 1,01 & 1,06 & 2,44 \\
\hline$S_{6}$ & 2,21 & 1,09 & 0,98 & 0,97 & 1,02 & 2,03 \\
\hline \multicolumn{7}{|c|}{$X_{I}=b / a=0,4, X_{2}=\alpha=0,22 \pi$} \\
\hline$S_{I}$ & 3,89 & 0,81 & 0,58 & 0,66 & 1,11 & 3,64 \\
\hline$S_{2}$ & 2,45 & 1,14 & 0,95 & 0,99 & 1,27 & 2,53 \\
\hline$S_{3}$ & 5,84 & 0,77 & 0,46 & 0,46 & 0,77 & 3,17 \\
\hline$S_{4}$ & 2,65 & 1,11 & 0,93 & 0,93 & 1,11 & 2,68 \\
\hline$S_{5}$ & 4,12 & 1,02 & 0,67 & 0,67 & 1,0 & 4,18 \\
\hline$S_{6}$ & 2,05 & 1,47 & 1,27 & 1,25 & 1,52 & 2,12 \\
\hline \multicolumn{7}{|c|}{$X_{I}=b / a=0,2, X_{2}=\alpha=0,33 \pi$} \\
\hline$S_{I}$ & 1,98 & 1,01 & 1,0 & 1,0 & 1,01 & 1,82 \\
\hline$S_{2}$ & 1,73 & 0,88 & 0,86 & 0,86 & 0,87 & 1,77 \\
\hline$S_{3}$ & 1,97 & 1,01 & 1,0 & 1,0 & 1,01 & 2 \\
\hline$S_{4}$ & 2,03 & 1,03 & 1,0 & 1,0 & 1,03 & 2,05 \\
\hline$S_{5}$ & 1,94 & 1,01 & 1,0 & 1,0 & 1,01 & 1,93 \\
\hline$S_{6}$ & 1,79 & 0,92 & 0,86 & 0,86 & 0,88 & 1,56 \\
\hline \multicolumn{7}{|c|}{$X_{I}=b / a=0,3, X_{2}=\alpha=0,33 \pi$} \\
\hline$S_{I}$ & 2,35 & 1,01 & 1,01 & 1,01 & 1,03 & 1,87 \\
\hline$S_{2}$ & 1,63 & 0,91 & 0,88 & 0,88 & 0,9 & 2,1 \\
\hline$S_{3}$ & 2,17 & 1,02 & 0,98 & 0,98 & 1,02 & 2,41 \\
\hline$S_{4}$ & 2,22 & 1,07 & 1,01 & 1,01 & 0,86 & 2,27 \\
\hline$S_{5}$ & 2,44 & 1,02 & 1,01 & 1,01 & 1,02 & 2,37 \\
\hline$S_{6}$ & 1,72 & 0,92 & 0,88 & 0,88 & 0,96 & 1,96 \\
\hline \multicolumn{7}{|c|}{$X_{I}=b / a=0,4, X_{2}=\alpha=0,33 \pi$} \\
\hline$S_{I}$ & 2,25 & 1,01 & 1,01 & 1,02 & 1,07 & 2,66 \\
\hline$S_{2}$ & 2,28 & 0,95 & 0,9 & 0,9 & 0,97 & 2,31 \\
\hline$S_{3}$ & 2,25 & 1,02 & 0,93 & 0,93 & 1,02 & 2,51 \\
\hline$S_{4}$ & 2,19 & 1,12 & 1,01 & 1,01 & 1,12 & 2,42 \\
\hline
\end{tabular}




\begin{tabular}{|c|c|c|c|c|c|c|}
\hline \multirow{2}{*}{$\begin{array}{c}\text { Code of } \\
\text { structure }\end{array}$} & \multicolumn{7}{|c|}{ Coordinates of measurement points in relative units } \\
\cline { 2 - 7 } & $\boldsymbol{0}$ & $\mathbf{0 , 2}$ & $\mathbf{0 , 4}$ & $\mathbf{0 , 6}$ & $\mathbf{0 , 8}$ & $\mathbf{1 , 0}$ \\
\hline$S_{5}$ & 2,73 & 1,02 & 1,01 & 1,01 & 1,02 & 2,78 \\
\hline$S_{6}$ & 1,89 & 1,03 & 0,92 & 0,91 & 0,96 & 1,78 \\
\hline \multicolumn{7}{|c|}{$X_{I}=b / a=0,2, X_{2}=\alpha=0,44 \pi$} \\
\hline$S_{1}$ & 1,32 & 1,0 & 1,0 & 1,0 & 1,0 & 1,27 \\
\hline$S_{2}$ & 1,13 & 0,86 & 0,86 & 0,86 & 0,86 & 1,14 \\
\hline$S_{3}$ & 1,37 & 1,0 & 1,0 & 1,0 & 1,0 & 1,34 \\
\hline$S_{4}$ & 1,33 & 1,01 & 1,0 & 1,0 & 1,01 & 1,32 \\
\hline$S_{5}$ & 1,29 & 1,0 & 1,0 & 1,0 & 1,0 & 1,3 \\
\hline$S_{6}$ & 1,17 & 0,87 & 0,86 & 0,86 & 0,87 & 1,21 \\
\hline \multicolumn{7}{|c|}{$X_{I}=b / a=0,3, X_{2}=\alpha=0,44 \pi$} \\
\hline$S_{1}$ & 1,75 & 1,0 & 1,0 & 1,0 & 1,01 & 1,67 \\
\hline$S_{2}$ & 1,44 & 0,87 & 0,86 & 0,86 & 0,87 & 1,21 \\
\hline$S_{3}$ & 1,38 & 1,01 & 1,0 & 1,0 & 1,01 & 1,4 \\
\hline$S_{4}$ & 1,35 & 1,02 & 1,0 & 1,0 & 1,02 & 1,39 \\
\hline$S_{5}$ & 1,65 & 1 & 1,0 & 1,0 & 1,0 & 1,65 \\
\hline$S_{6}$ & 1,24 & 0,88 & 0,86 & 0,86 & 0,87 & 1,33 \\
\hline \multicolumn{7}{|c|}{$X_{I}=b / a=0,4, X_{2}=\alpha=0,44 \pi$} \\
\hline$S_{1}$ & 1,49 & 1,74 & 1,0 & 1,0 & 1,02 & 1,64 \\
\hline$S_{2}$ & 1,53 & 0,87 & 0,87 & 0,87 & 0,88 & 1,51 \\
\hline$S_{3}$ & 1,39 & 1,01 & 0,99 & 0,99 & 1,01 & 1,42 \\
\hline$S_{4}$ & 1,39 & 1,04 & 1,0 & 1,0 & 1,04 & 1,45 \\
\hline$S_{5}$ & 1,56 & 1,0 & 1,0 & 1,0 & 1,0 & 1,71 \\
\hline$S_{6}$ & 1,15 & 0,89 & 0,87 & 0,87 & 0,87 & 1,24 \\
\hline \multicolumn{7}{|c|}{1,0} \\
\hline
\end{tabular}

inhomogeneity of field were observed for the $S_{2}, S_{4}$ and $S_{6}$ structures, maximum value of inhomogeneity coefficient $k$ was not exceeding 2,68. At $X_{2}=\alpha=0,22 \pi$, the $S_{5}$ structure with maximum value of coefficient $k=2,73 \ldots 2,78$ should be considered as rational.

Thus, an approach for estimation of inhomogeneity degree of magnetic field in polygradient medium of synthesized structures by comparing of inhomogeneity coefficient along characteristic lines has been proposed and implemented. The disadvantage of this approach is the dependence of choice of characteristic lines from location of zones of field inhomogeneity in working gaps.

\section{CONCLUSION}

In the paper the indicator of field inhomogeneity was used for choice of the rational structure of polygradient medium and geometric dimensions of its plate elements. The results of research showed that the highest inhomogeneity degree of field was in synthesized structures of polygradient medium of separators at $X_{2}=\alpha=0,22 \pi ; 0,33 \pi$. For relatively large air gaps $\left(X_{1}=b / a=0,2\right)$ and at $X_{2}=\alpha=0,22 \pi ; 0,33 \pi$, rational should be considered the $S_{4}$ structure, as it provides the highest inhomogeneity degree of field. At minimum air gaps $\left(X_{1}=b / a=0,4 ; X_{2}=\alpha=0,22 \pi\right)$, the $S_{5}$ structure provides the highest inhomogeneity degree of field. The received results can be recommended for practical use with purpose of preliminary estimation of efficiency of structures synthesis of separators polygradient matrix and choice of rational structure. The direction of further research is the analysis of inhomogeneity of magnetic field in polygradient medium by estimating of inhomogeneity spectrum of field in plane of working zone. This will allow us to receive a generalized indicator of field inhomogeneity for each of structures.

\section{REFERENCES}

With reduction of interpolar air gap $\delta$ at $X_{I}=b / a=0,3$, the highest value of inhomogeneity index of field $k$ in zone that close to teeth was observed in the $S_{1}$ and $S_{5}$ structures. These structures are also characterized by a high intensity of the magnetic field in zone between teeth, where the field approaches to homogeneous field $(k \sim 1)$. At $X_{2}=\alpha=0,22 \pi$, it is preferable to use the $S_{1}$ structure with maximum value of coefficient $k$ in zone that close to teeth equals to $2,62 \ldots 2,69$. At $X_{2}=\alpha=0,33 \pi$, the best characteristics of inhomogeneity of field shows the $S_{5}$ structure with maximum value of the coefficient $k=2,37 \ldots 2,44$. With a further reduction of interpolar air gap $\delta\left(X_{1}=b / a=0,4\right)$ at $X_{2}=\alpha=0,22 \pi$, a significant increase of inhomogeneity degree of magnetic field in zone that close to teeth was observed. For example, for the $S_{3}$ structure, coefficient $k$ near the tooth was 5,84, and for the $S_{5}$ structure it was 4,18. However, for $S_{3}$ and $S_{5}$, significant weakening of magnetic field in zone between teeth was characteristically, therefore, these structures can not be considered rational. At $X_{2}=\alpha=0,22 \pi$, the best characteristics of

[1] J. Svoboda, Magnetic Methods for the Treatment of minerals. Amsterdam; New York: Elsevier, 1987.

[2] I. Soloshych and I. Shvedchykova, "Development of systematlics ranked structure of environmental protecting equipment for cleaning of gas emissions, wastewater and solid waste," Eastern-European Journal of Enterprise Technologies, no. 6/10 ( 84 ), pp. 17-23, 2016.

[3] H. Chen, D. Bockenfeld, D. Rempfer, M. D. Kaminski, X. Liu and A. J. Rosengart, "3-D analysis of a high gradient magnetic separator for biomedical applications," Journal of Magnetism and Magnetic Materials, vol. 320, pp. 279-284, 2008.

[4] L. Ren, Sh. Zeng and Y. Zhang, "Magnetic field characteristics analysis of a single assembled magnetic medium using ANSYS software," International Journal of Mining Science and Technology, no. 25, pp. 479-487, 2015.

[5] L. Chen, G. Xu, Sh. Wen, S. Liu and L. Gao, "Arrangement in Matrix on High Gradient Magnetic Separation Performance," Advanced Materials Research, vol. 634-638, pp. 3351-3354, 2013.

[6] I. A. Shvedchikova, I. A. Lutsenko and J. A. Romanchenko, "A study of polygradient media struture regularities," Eastern-European Journal of Enterprise Technologies, vol. 4, no. 7 ( 76 ), pp. 62-67, 2015.

[7] N. A. Nikolov, "Quantitative criterion of the spatial heterogenety of electromagnetic field in the near-field zone of a loop radiator," Kibernetika $i$ systemny analiz, no. 2, pp. 170-177, 2013. 